\title{
Redimensionamento de redes de telefones públicos urbanos
}

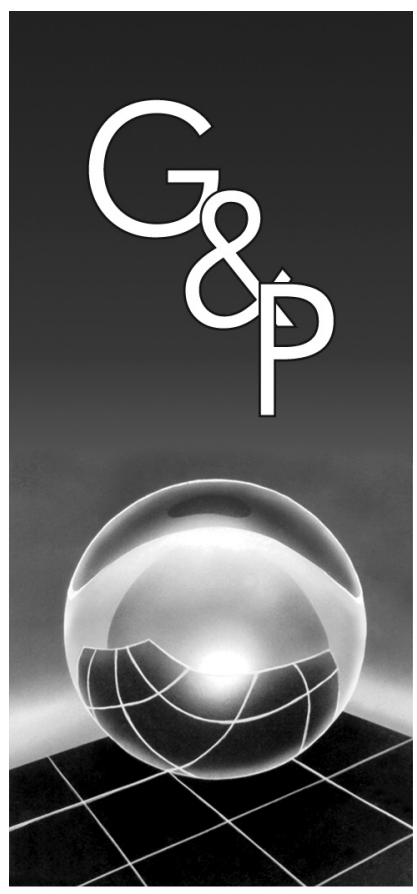

\author{
Leonardo Junqueira Lustosa \\ Cristina de las Nieves Araneda-Fuentes \\ Daniel de Mello Schaefer
}

\section{Resumo}

Este artigo discute o problema de redimensionamento de uma rede de telefones públicos urbanos visando melhorar a lucratividade do negócio sujeito a exigências da Agência Nacional de Telecomunicações. O estudo tem um viés pragmático, dando ênfase à obtenção de uma boa solução usando meios ao alcance de empresas. Peculiaridades do problema conduzem à formulação de um modelo de localização e atribuição com cobertura mínima que, em escala real, assume um porte que inviabiliza sua solução exata por métodos convencionais de programação inteira mista. É, então, examinada a viabilidade de se obter uma boa solução sem recurso a métodos complexos e de difícil implementação. Discutem-se formas de redução e particionamento do problema, sua solução por interrupção prematura de algoritmo de ramificação e limitação (branch-and-bound) e, também, por meio de uma heurística simples e de fácil implementação. São apresentados os resultados de um ensaio computacional que indicam a viabilidade de aplicação do modelo no ambiente industrial.

Palavras-chave: Telefonia pública. Programação linear inteira. Localização-atribuição. Cobertura mínima. Heurística.

\section{Introdução}

Com a privatização do setor de telefonia em 1997, as operadoras concessionárias de telefonia fixa passaram a ser reguladas pela Agência Nacional de Telecomunicações, que impôs exigências relativas à instalação de telefones públicos em áreas urbanas e rurais (BRASIL, 1998). Sendo a operação de telefones públicos (telefone de uso público - TUP) considerada uma atividade de baixa lucratividade e de importância estratégica declinante devido à forte concorrência da telefonia móvel, é do interesse das operadoras rever o número e a localização dos seus aparelhos. Estando as concessionárias de telefonia fixa sujeitas à obrigação de manter o serviço de telefonia pública, é surpreendente que, fora a menção de um estudo de propriedade privada (WORKSHOP DE PARCERIA UNICAMP \& TELEFÔNICA, FASE II, 2004), apenas um trabalho sobre o assunto (SCHAEFER, 2006) tenha sido encontrado na bibliografia. O problema tratado é similar, em natureza gerencial e em estrutura matemática, aos problemas de unificação de redes de distribuição (caixas eletrônicos, postos de distribuição de gás, entre outros) quando empresas passam a operar de forma conjunta, seja por acordos comerciais, ou por fusões e aquisições.
Como esse negócio já apresentou lucratividade mais atraente, o número de TUPs instalados geralmente excede as exigências da ANATEL que, na área urbana, podem ser expressas por dois parâmetros, a saber: (a) a distância de cada possível usuário ao TUP mais próximo não deve exceder 300 metros; e (b) o sistema deve oferecer um mínimo de três TUPs por 1.000 habitantes. Portanto, mais do que decidir sobre onde localizar novos terminais telefônicos, o problema é, sobretudo, decidir que terminais devem ser mantidos e quais os que deverão ser removidos. O problema de localização de novos telefones parece ser bem mais complexo porque depende de informações detalhadas que, em última análise, irão determinar a lucratividade do terminal de TUP. Dentre essas informações estão o custo de estender o cabeamento, a exposição a intempéries e ao vandalismo, além, é claro, da conveniência para os potenciais usuários.

Além de sugerir uma solução para um problema pouco explorado e relevante para empresas de telefonia fixa, este artigo tem o propósito de contribuir para a disseminação do uso industrial da programação matemática com mais um caso em que a análise e a formulação cuida- 
dosas levaram a resultados satisfatórios com recursos ao alcance da empresa. $\mathrm{O}$ estudo aqui relatado tem um viés pragmático e o objetivo de propor um modelo de programação matemática de utilização viável em empresas que mantenham rede de TUPs. Por isso, procura avaliar a viabilidade computacional do modelo em escala real sem recurso a métodos de solução complexos, especializados e de implementação difícil.

O texto a seguir se estrutura em seis seções, sendo a primeira esta introdução. A segunda seção apresenta o problema, discutindo suas principais características e fazendo referência à bibliografia diretamente relevante. A Seção 3 apresenta um modelo de programação linear inteira mista, desenvolvido para auxiliar o estudo de redimensionamento da rede, e comenta diversos aspectos de ordem prática considerados na sua formulação. A Seção 4 discute a dificuldade computacional decorrente do grande porte que o modelo assume em situações reais e propõe formas simples para obtenção de uma boa solução. Dentre essas propostas, estão formas de redução e particionamento do problema, bem como uma heurística míope baseada na estratégia de fechamentos sucessivos (drop strategy). Resultados de testes computacionais levados a cabo para verificar a viabilidade de aplicação prática do modelo são apresentados na Seção 5. Finalmente, na Seção 6, são feitas observações de caráter geral, apresentadas algumas conclusões mais importantes da pesquisa e indicados alguns pontos que ainda necessitam de maior desenvolvimento para uma aplicação industrial.

\section{0 problema}

Em função de sua história de monopólio de governo e posteriormente de concessão pública, a telefonia fixa no Brasil, em particular o serviço de telefones públicos, apresenta-se como um negócio pouco atraente numa indústria de grande dinamismo (SCHAEFER, 2006). Além disso, é de se esperar que a lucratividade desse negócio esteja em declínio devido à crescente popularidade do telefone móvel. Assim, a manutenção de uma rede de telefones públicos é vista apenas como uma contrapartida social que as concessionárias de telefonia fixa estão obrigadas a oferecer para ter acesso a negócios bem mais lucrativos. Essa baixa atratividade tem sido atribuída, em grande parte, às normas estabelecidas pela agência reguladora, o que, aparentemente, leva as empresas a tratar a questão nos seus aspectos de política pública, dando pouca atenção às possibilidades de melhoria do negócio. Indicação disso é que, algumas empresas, preocupadas em atender rapidamente às condições impostas pela ANATEL para ter acesso a outros negócios mais atraentes e estratégicos, ampliaram sua rede pública sem muito cuidado com a lucratividade. Diante da ineficiência atual de suas redes e da impossibilidade de abandoná-las, é de interesse das empresas modificá-las para aumentar a lucratividade do serviço (mantendo a qualidade e disponibilidade exigidas) e, também, usá-lo para criar uma imagem positiva junto ao público. Por isso, além de atender às exigências de cobertura e de densidade mínima acima mencionadas, um limite deve ser imposto à demanda em cada TUP para evitar congestionamentos que podem trazer transtornos aos usuários.

Uma informação fundamental para o problema é, obviamente, a distribuição geográfica da demanda e, dado o contexto do problema, algumas considerações devem ser feitas. Em primeiro lugar, há que se considerar que o único dado concreto e imediatamente disponível de demanda é o faturamento em cada TUP na configuração atual do sistema. Uma determinação mais precisa da distribuição geográfica da demanda potencial poderia ser tentada através de algum modelo descritivo do espalhamento da demanda em torno dos TUPs existentes e, posteriormente, concentrada em pontos discretos (DREZNER; DREZNER, 1997). Entretanto, mesmo que dados sobre a distribuição espacial da demanda potencial pudessem ser levantados, sem dados sobre as condições de acesso a cada TUP, tal modelo continuaria sendo uma aproximação grosseira da distribuição da demanda real. Em um estudo envolvendo toda a rede, a obtenção de dados de acessibilidade é inviável do ponto de vista prático, pois exigiria um trabalho que considerasse as vias e equipamentos urbanos, a localização de estabelecimentos comerciais e outros detalhes. Por isso, aqui, a demanda em cada TUP será considerada igual ao seu faturamento.

Em segundo lugar, observa-se que a demanda em um TUP não é necessariamente gerada por usuários localizados muito próximos ao TUP, mas sim pelos usuários numa "área de influência" ou "área de cobertura" do TUP. Dessa forma, se os TUPs inicialmente instalados atendem à restrição de distância máxima, não se pode garantir que ao se remover um dado TUP, que esteja a uma distância menor do que 300 metros de outro, a exigência de distância máxima será atendida para todos os usuários do TUP retirado. Entretanto, essas eventuais falhas de cobertura são facilmente detectadas por visualização em um sistema de informação geográfica e podem ser corrigidas.

Finalmente, outro aspecto que diz respeito à demanda é o que ocorre quando um TUP é retirado. Fica sua demanda completamente perdida ou é integralmente, ou parcialmente, transferida para um ou mais TUPs próximos não congestionados? A literatura apresenta propostas para resolver o problema da distribuição da demanda entre postos de serviço (PERALES, 2002; MARIANOV; SERRA, 1998). A determinação precisa da redistribuição da demanda de um TUP retirado envolve, além da distância dele para os TUPs dentro de sua área de cobertura, o nível de utilização desses outros TUPs. É, portanto, um problema de equilíbrio complexo demais 
para solução neste contexto e, além disso, os dados que ela exigiria são de obtenção inviável no caso em questão. Por isso, uma modelagem aproximada se justifica e permanece útil para avaliação do potencial de ganho, assim como para decisões de nível tático. Neste trabalho, como será visto adiante, a hipótese feita é que parte da demanda do TUP retirado é transferida para outro(s) TUP(s) próximo(s) em função apenas da distância.

Consideração importante é a confiabilidade do serviço. Telefones públicos são equipamentos muito expostos a intempéries e, principalmente, a vandalismo e ações maliciosas para evitar a correta tarifação. Tornar tais equipamentos mais resistentes a essas ameaças é sempre uma questão de custo-benefício cujo equilíbrio resulta em equipamentos ainda muito sujeitos à interrupção de funcionamento. Por isso, a confiabilidade do serviço é uma questão importante. $\mathrm{O}$ aumento da confiabilidade do serviço pode ser obtido através de redundância, ou seja, dispondo os terminais de tal forma que, na eventualidade de falha de um terminal, exista outro a uma distância ainda aceitável. Encontram-se na literatura diversos modelos para localização de unidades de serviço de alta exigência de disponibilidade, como ambulâncias e equipamentos de combate a incêndios (HOGAN; REVELLE, 1986; FIGUEIREDO et al., 2003; GALVÃO et al., 2005). De novo, a consideração desse detalhe adicional pode ser interessante, mas o ponto de vista essencialmente pragmático deste trabalho não recomenda sua incorporação antes que a prática demonstre que a complexidade adicional que ele traz é, de fato, justificada pela necessidade.

$\mathrm{O}$ modelo a seguir foi desenvolvido tendo em mente decisões de nível tático como identificar áreas e unidades críticas para a lucratividade, ou o potencial de ganho, sob diferentes cenários de declínio de demanda. Para decisões operacionais, o modelo permite gerar uma proposta inicial de remoções a ser revista manualmente através do sistema de informações geográficas e de inspeções locais. Isso porque outras considerações são necessárias para decisões finais relativas à remoção de TUPs como, por exemplo, o estado de conservação do TUP ou o grau de sua exposição a ações de vandalismo. Para esse nível de detalhe, um sistema de informações geográficas, o conhecimento tácito dos funcionários que lidam com os diversos aspectos envolvidos e as inspeções in loco são mais adequados do que qualquer modelo de otimização.

\section{0 modelo}

Seja uma rede de $n$ TUPs instalados com as características abaixo.

- $D(i)$ - demanda no TUP $i$ representada pela sua receita mensal;

- $C U S T(i)$ - custo de manutenção mensal do TUP $i$;
- MAXCAP $(i)$ - capacidade máxima de atendimento do TUP i (medida em receita já que esta é praticamente proporcional ao tempo de utilização);

- $\operatorname{DIST}(i, j)$ - distância do TUP $i$ ao TUP $j$;

- $A D M T(i, j)$ - admitância de $j$ para $i$, ou seja, fator de redução da demanda do TUP $j$ que chegará ao TUP $i$ caso se decida remover o TUP $j$. É função decrescente de $D I S T(i, j)$;

- MAXDIST - distância máxima permitida para que um TUP possa atender à demanda de outro;

- MINTUPS - número mínimo de TUPs exigido.

Definem-se então os seguintes conjuntos e variáveis:

- $A$ - conjunto de todos os índices de TUPs, $i=1, \ldots n$;

- $A_{\mathrm{i}}$ - domínio de cobertura do TUP $i$, ou seja, o conjunto de todos os índices $j$ tais que $\operatorname{DIST}(i, j)<$ MAXDIST;

- $X(i, j)$ - fração da demanda do TUP $j$ dirigida para o TUP $i$ (caso ele esteja na cobertura do TUP $j$ );

- $Y(i)$ - variável binária indicadora de remoção do TUP $i$ ( 0 se o TUP é removido e 1 se mantido).

No modelo, $A D M T(i, j)$ é o fator de redução da demanda que é transferida do TUP $j$ para o TUP $i$, portanto, a demanda que o TUP $i$ efetivamente recebe do TUP $j$ é $D(j) X(i, j) A D M T(i, j)$. A função de admitância, $A D M T(i, j)$, deveria ser definida a partir de uma análise estatística dos dados históricos de remoções ou de algum estudo empírico sobre a distância percebida pelos usuários. Drezner et al. (1998) propõem uma função logística para a admitância e a justificam em termos de um modelo probabilístico de escolha.

O modelo localização-atribuição com exigência de cobertura é proposto conforme as Equações 1 a 6 .

$$
\begin{aligned}
& \text { Maximizar } \sum_{i \in A} \sum_{j \in A_{i}} \\
& {[D(j) A D M T(i, j) X(i, j)-C U S T(i) Y(i)]}
\end{aligned}
$$

S. a

$$
\sum_{i \in A_{j}} X(i, j)=1, \forall j \in A
$$

$$
\begin{aligned}
& \operatorname{MAXCAP(i)Y(i)-} \\
& -\sum_{j \in A_{i}} D(j) A D M T(i, j) X(i, j) \geq 0, \forall i \in A
\end{aligned}
$$

$$
\sum_{j \in A} Y(j) \geq M I N T U P S
$$

$$
\begin{gathered}
X(i, j) \geq 0, \quad \forall(i, j): i \in A, j \in A_{i} \\
Y(i) \in\{0,1\}, \quad \forall i \in A
\end{gathered}
$$

A função objetiva (1) representa o lucro mensal bruto obtido na operação. As restrições (2) exigem que a 
demanda de cada TUP seja integralmente atendida por ele mesmo ou (com perda) por outros TUPs dentro da sua área de cobertura, garantindo que um TUP só será removido se houver outro dentro do padrão de cobertura. As restrições do grupo (3) exigem que, para atender a alguma demanda, o TUP deverá estar disponível e, nesse caso, o total da demanda a que ele atende não pode exceder sua capacidade. Como demanda e utilização são proporcionais, apenas demandas são consideradas no modelo. A capacidade máxima de um TUP pode ser estimada pela receita máxima observada. Conforme a sugestão de Marianov e Serra (1998), a capacidade poderia, também, ser determinada de outras formas como, por exemplo, limitando-se a probabilidade de o comprimento da fila, ou o tempo na fila de cada TUP, superar um dado valor. A restrição (4) requer que o número de TUPs mantidos não seja inferior ao mínimo de TUPs exigido pela ANATEL. As condições de não-negatividade das variáveis $X$ e as de que as variáveis $Y$ assumam apenas os valores 0 ou 1 são impostas pelos grupos (5) e (6), respectivamente. Note-se que, em virtude das restrições (2) e (3), as variáveis $X(i, j)$ só podem assumir os valores positivos se o TUP $i$ não tiver sido removido.

Em princípio, as distâncias deveriam ser as efetivamente percorridas, mas estas não estão disponíveis e teriam que ser penosamente levantadas. O uso de distância em quarteirão (ou Manhattan) seria uma boa aproximação, mas a irregularidade de orientação dos quarteirões cria problemas. Uma aproximação para a distância Manhattan pode ser feita usando-se a distância euclidiana e exigindo-se que o padrão de cobertura seja $1 / \sqrt{2}$ da distância máxima percorrida admitida pela ANATEL. Dessa forma, desde que as vias se cruzem ortogonalmente, qualquer que seja a orientação do arruamento, a exigência da ANATEL será sempre atendida (exatamente ou com alguma folga).

Conforme mencionado acima, quando um TUP é retirado, é de se esperar que uma parte da sua demanda se transfira para um ou mais dos TUPs restantes mais próximos. Na realidade, essa transferência parcial da demanda de um TUP removido para os remanescentes tem dois efeitos importantes. O primeiro é a perda de receita referente à parcela de demanda não transferida, $\mathrm{o}$ segundo é a carga de serviço que a parcela transferida traz para o TUP receptor da demanda. Para o primeiro efeito, o que importa é apenas a parcela da demanda que é transferida (efeito na função objetiva), mas para o segundo é relevante determinar para que TUP (ou TUPs) a demanda é transferida (efeito nas restrições (3)).

Para modelar essa transferência, é razoável considerar que, quando a demanda de um TUP retirado se transfere para outro, ocorre uma perda que é função crescente da distância entre o TUP removido e o TUP receptor. Na realidade, essa perda deve ser, também, função do nível de saturação da capacidade no TUP receptor, pois o usuário pode desistir ou escolher outro TUP se tiver que enfrentar uma fila. A consideração do nível de saturação dos TUPs receptores no modelo exigiria determinar uma condição de equilíbrio complexa cada vez que um TUP fosse removido. Para manter o problema tratável, no modelo acima se supõe que a demanda de um TUP removido é transferida para o TUP mais próximo (i.e., com maior admitância) até esgotar a capacidade deste TUP; se ainda restar demanda a ser transferida, ela irá para o seguinte mais próximo, e assim por diante, até que toda a demanda do removido seja transferida para TUPs em sua área de cobertura, conforme exigem as restrições (2) e (3). De acordo com a função objetiva e com as restrições do grupo (3), apenas parte da demanda do TUP retirado, de fato, chega ao(s) TUP(s) receptor(es).

Na maioria dos problemas de localização-atribuição com exigência de cobertura, a função objetiva é maximizar a população coberta pelas instalações prestadoras de serviços ou minimizar o número de instalações para uma dada cobertura (BRANDEAU; CHIU, 1989; HALE; MOBERG, 2003). A maximização de lucro no modelo (1)-(6) foge a esse padrão e o coloca entre os raros problemas de localização deste tipo encontrados na literatura. Um outro aspecto distintivo é o fato de não haver exigência de integralidade para as variáveis de alocação $X$, ou seja, permite-se que um ponto de demanda seja atendido por mais de uma instalação de serviços (neste caso, um TUP).

O modelo proposto apresenta uma estrutura simples com matriz esparsa e pequenos grupos de variáveis bem conectadas. Com essas características, seria um problema computacionalmente fácil de resolver por métodos exatos convencionais, não fosse seu grande porte. Em casos reais, o problema tem algo como dezenas de milhares de variáveis $X$, milhares de variáveis $Y$ e milhares de restrições dos tipos (2) e (3). Por isso, é importante abordar as questões de redução do esforço computacional e de viabilidade da resolução de problemas de porte real.

\section{Redução do esforço computacional}

Algumas possibilidades de redução do porte do problema através de seu particionamento em subproblemas computacionalmente mais tratáveis são discutidas abaixo, seguidas de formas de obter soluções aproximadas (para o problema inteiro ou particionado).

\subsection{Redução e recomposição do problema}

$\mathrm{O}$ porte do modelo pode ser reduzido examinando-se detalhes dos dados conforme as seguintes proposições, todas bem intuitivas e fáceis de serem demonstradas com base no próprio modelo.

Proposição 1 - fixação de telefones de uso público (TUPs) isolados. 
"Qualquer TUP $r$ 'isolado', i.e., $A_{\mathrm{r}}=\{r\}$, com $r \in \mathrm{A}$, será mantido na solução ótima, podendo, portanto, ser eliminado de consideração na solução do problema e descontado do número mínimo de TUPs exigido."

Proposição 2 - separação natural (problemas quase disjuntos).

"Se o conjunto $A$ de todos TUPs puder ser particionado em $K$ subconjuntos disjuntos de TUPs, digamos $S_{\mathrm{k}}, k=1, \ldots$, $K, K<\operatorname{card}(A)$, tais que $\bigcup_{k=1}^{K} S_{k}=A, k \neq h \Rightarrow S_{k} \cap S_{h}=\varnothing \mathrm{e}$ que, para quaisquer $p \in S_{i}$ e $r \in S_{j}, i \neq j \Rightarrow A_{p} \subseteq S_{i}, A_{r} \subseteq S_{j}$ e $A_{p} \cap A_{r}=\varnothing$, então o problema pode ser decomposto em $K$ subproblemas (cada um considerando um subconjunto $S_{\mathrm{k}}$ ) ligados apenas pela restrição (4)."

Proposição 3 - fixação de telefones de uso público (TUPs) saturados.

"Se todos os TUPs tiverem a mesma capacidade máxima e o mesmo custo de manutenção mensal, então um TUP que esteja sem folga de capacidade deve ser mantido na solução ótima."

O problema real pode ser reduzido através das proposições 1 e 3, mas, mesmo com essas reduções, um problema real típico permaneceria com porte computacionalmente inviável. A Proposição 2 permite o particionamento do problema original em subproblemas ligados apenas pela restrição (4). Entretanto, manter essa restrição de ligação, mesmo em um eventual esquema de decomposição, não ajudaria muito porque é sabido que tais esquemas podem ter uma convergência lenta. Portanto, do ponto de vista prático, a decomposição pela Proposição 2 só seria realmente interessante no caso de a restrição (4) não ser ativa na solução ótima ou se a empresa tiver critérios que indiquem qual o número mínimo de TUPs que se deve ter em cada uma das áreas disjuntas. Mesmo que não existam áreas disjuntas, é possível fazer um particionamento em áreas delimitadas por bordas de baixa densidade de TUPs e, posteriormente, tratar caso a caso os TUPs de fronteira. Esse procedimento deverá levar a uma boa aproximação porque a influência de um TUP sobre outros é rapidamente decrescente com a distância.

\subsection{Resolução aproximada}

Como frequentemente a convergência inicial nesse tipo de problema é relativamente rápida, deverá ser possível obter boas soluções em tempo satisfatório usando-se um otimizador genérico para programação linear inteira e interrompendo-se prematuramente a execução. Tal interrupção seria feita quando a brecha de otimalidade (optimality gap) atingisse um dado valor, ou seja, terminando a execução quando o algoritmo gerasse uma solução inteira com valor não inferior a uma porcentagem do limite superior gerado por relaxação da integralidade. Mesmo assim, é possível que essa tentativa só tenha sucesso se o problema for decomposto em subproblemas como esboçado acima.

\subsection{Método heurístico}

O método heurístico é baseado na idéia de remoções sucessivas (drop strategy) segundo uma análise míope do aumento de lucratividade obtido a cada remoção. Assim, tenta-se remover sucessivamente o TUP (ou um dos TUPs) que apresente o menor lucro até que todos os TUPs tenham sido examinados, ou o número mínimo de TUPs tenha sido atingido. O TUP em exame é mantido se for necessário para a cobertura exigida ou se sua remoção implicar em redução do lucro total; caso contrário, o TUP é definitivamente removido.

Além das definições dos elementos do modelo feitas acima, para descrever o procedimento, são necessários alguns elementos adicionais definidos, conforme se segue:

ATestar - conjunto de índices dos TUPs ainda não examinados;

LucroTotal - valor da função objetivo (1) para o último ótimo do problema (1)-(5) obtido com atual fixação das variáveis $Y($.$) ;$

ÚltimoLucro - variável auxiliar;

Atual - variável auxiliar indicando o TUP em exame; LucroTup(i) - lucro do TUP i,

$$
\sum_{j \in A_{i}}[D(j) A D M T(i, j) X(i, j)-C U S T(i) Y(i)] .
$$

No procedimento, a instrução "ATestar :=ATestar - Atual" indica a remoção do elemento "Atual" do conjunto ATestar.

Procedimento Heurístico:

ATestar := A;

Resolva Problema de programação linear (1) - (5) com $\mathrm{Y}(\mathrm{i}):=1, \forall \mathrm{i} \in \mathrm{A}$;

0] Enquanto $\left[\sum_{\mathrm{i} \mathrm{A} A} Y(i)>\operatorname{MINTUPS}\right.$ e $\operatorname{Card}($ ATestar $)>$ $\forall \mathrm{i} \in \mathrm{A}$ Testar;

Atual := $r$ tal que LucroTup(r) $<$ LucroTup(i),

$Y($ Atual $):=0$;

Resolva Problema de programação linear (1)-(5) com as variáveis $Y(\cdot)$ fixadas nos valores atualmente atribuídos;

Se Problema (1)-(5) for infactível

Então

$$
Y(\text { Atual }):=1 ;
$$

Senão

Se LucroTotal < ÚltimoLucro

Então

$$
Y(\text { Atual }):=1
$$

Senão

FimSe

ÚltimoLucro := LucroTotal; FimSe

ATestar := ATestar - Atual;

FimEnquanto

Resolva Problema (1)-(5) com as variáveis $Y(\cdot)$ fixadas nos valores atualmente atribuídos

Fim Procedimento Heurístico. 
Note-se que, antes de encerrar o procedimento, o modelo é reotimizado para gerar a solução final que não será a última obtida se o último teste indicar infactibilidade ou redução no lucro total. Essa reotimização poderia ser evitada, armazenando-se não só o valor ÚltimoLucro, mas também a solução que o gerou. Como o tempo para essa reotimização é pequeno, a simplicidade de implementação no software utilizado levou à escolha da forma acima.

\section{Viabilidade computacional}

O esforço computacional para resolver o problema depende do número de TUPs e da cardinalidade dos domínios dos TUPs que, por sua vez, são determinados pela distribuição espacial e pelo padrão de cobertura. Por isso, o teste de viabilidade prática do método de solução proposto deve ser feito com dados que sejam típicos de situações reais.

O problema em escala real usado para os testes refere-se a uma área urbana de 505.400 habitantes (exigindo, portanto, um mínimo de 1.517 TUPs instalados) com 4.099 TUPs atualmente instalados (13 deles eliminados nos testes por problemas com dados do cadastro). Os dados foram fornecidos por uma empresa e alterados por motivos de confidencialidade, mantendo as características mais relevantes para os testes. Além do padrão de cobertura, essas características incluem o total de TUPs e, também, sua distribuição geográfica, capacidade e relação custo/receita. A Figura 1 dá uma idéia do espalhamento dos TUPs na área estudada. Deve-se aqui ressaltar que muitos dos pontos da figura correspondem a dois ou mais TUPs muito próximos.

$\mathrm{Na}$ falta de informações para uma definição precisa e por se tratar de um valor sem impacto no porte do problema, a função de admitância foi arbitrariamente definida como $\operatorname{ADMT}(i, j)=1 /[1+0,004 \operatorname{DIST}(i, j)]$ (distâncias medidas

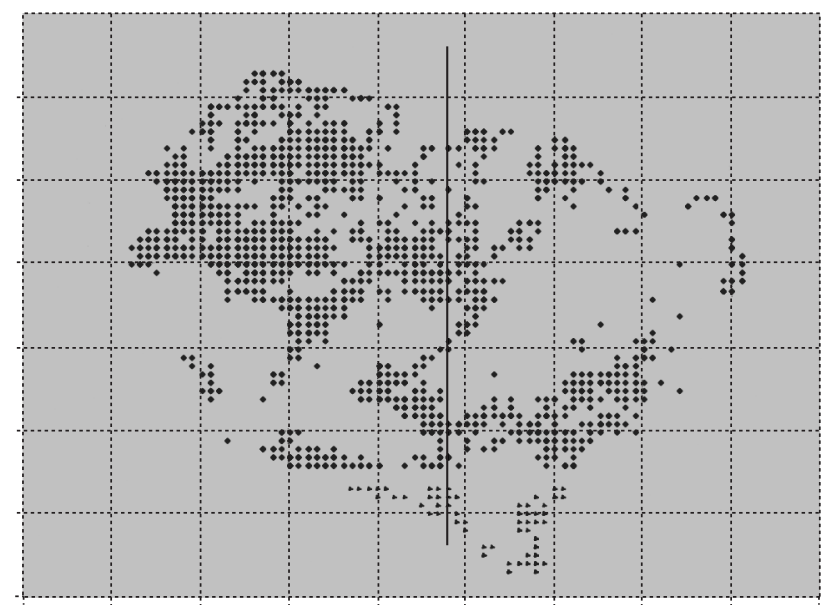

Figura 1. Aspecto da localização dos TUPs na área de estudo. em metros) o que gera perdas que estes autores julgaram razoáveis. Devido à pouca importância da admitância para os testes, a distância $\operatorname{DIST}(i, j)$ foi tomada como sendo a distância euclidiana simplesmente. Entretanto, numa aplicação real, seria recomendável fazer a correção de distância sugerida por Drezner e Drezner (1997) para compensar o fato de a demanda não se encontrar concentrada no exato local do TUP. Com isso, a distância corrigida $d^{\prime}$ seria dada por $d^{\prime}=\sqrt{d^{2}+0,24 A}$, em que $d$ é a distância real e $A$, a área por onde se distribui a demanda. $O$ padrão de cobertura foi estabelecido como MAXDIST $=300 / \sqrt{2}=212,2 \mathrm{~m}$, que equivale a uma distância máxima de $300 \mathrm{~m}$ em geometria de quarteirão.

Para testar a viabilidade de se fazer uma decomposição geográfica, a área foi particionada em duas (leste e oeste) segundo uma linha norte-sul (vertical na Figura 1), buscando equilibrar o número de TUPs em cada uma delas e definir bordas com poucos TUPs.

Os testes feitos tiveram como objetivo avaliar os seguintes aspectos:

- a qualidade e o tempo de resolução do problema pelo procedimento heurístico e pelo método de ramificação e limitação $(\mathrm{B} \& \mathrm{~B})$ com interrupção prematura; e

- o efeito da estratégia de decomposição por particionamento geográfico.

Em face desses objetivos, foram realizadas as seguintes corridas:

a) Sobre a área total (toda a área da Figura 1):

- obtenção de uma solução do problema (com e sem a restrição de número mínimo de TUPs) pelo método heurístico; e

- obtenção de uma solução do problema (com e sem a restrição de número mínimo de TUPs) usando o $\mathrm{B} \& \mathrm{~B}$, com interrupção prematura decorrido o mesmo tempo de processamento tomado pelo método heurístico. Tentou-se obter solução comprovadamente ótima pelo B\&B, mas o otimizador terminou anormalmente por esgotamento da pilha de nós abertos após mais de 24 horas de processamento. Talvez uma solução ótima pudesse ser obtida com parametrização adequada do otimizador, ou com o emprego de otimizador mais eficiente, como o CPLEX.

b) Sobre cada uma das partes da área particionada (leste e oeste):

- obtenção de uma solução para o problema de cada parte sem a restrição de número mínimo de TUPs pelo método heurístico; e

- obtenção de uma solução de forma análoga à do segundo ponto acima.

O modelo foi implementado no ambiente AIMMS 3.6 (BISSCHOP; ROELOFS, 1999) usando o otimizador XA-14 e executado em computador com processador 
Pentium 4 - $3.00 \mathrm{GHz}$ e 1Gbyte de RAM sobre um sistema operacional Windows XP.

\subsection{Resultados para toda a área}

A Tabela 1, abaixo, apresenta os resultados da solução do problema, respectivamente, sem e com restrição de número mínimo de TUPs, na parte superior da tabela por $\mathrm{B} \& \mathrm{~B}$ e na inferior pelo método heurístico. Para permitir comparação, também foi registrada uma solução factível obtida pelo $\mathrm{B} \& \mathrm{~B}$ em um tempo similar ao tempo tomado pela heurística para gerar a sua solução final. Observa-se que os tempos tomados pela heurística são viáveis para um estudo em escala real. Isso porque, em função da desejada simplicidade da abordagem, não foram esgotadas as possibilidades de redução do problema, usando-se as proposições 1 e 3 , ou de melhoria no desempenho do algoritmo de B\&B usado pelo otimizador.

A superioridade da solução pela heurística sobre o B\&B é patente em ambos os casos, isto é, com e sem a restrição de um número mínimo de TUPs. No problema com limite de número de TUPs, os gaps foram inferiores a $1,5 \%$, sugerindo que o $\mathrm{B} \& \mathrm{~B}$, com terminação prematura (como, por exemplo, no tempo que toma a heurística para gerar a sua solução final), é capaz de gerar soluções satisfatórias para aplicações reais. Não obstante, enquanto a heurística encontra uma solução ótima ( $g a p$ de 0\%), o algoritmo B\&B gera, em tempo similar (3 horas e 7 minutos), uma solução com um gap de 1,44\% que, daí em diante, cai lentamente até $0,23 \%$ na solução factível encontrada depois de 24 horas.

Apesar de a heurística também ter tido melhor desempenho no problema sem limite para o número de TUPs, a superioridade é menos marcante. Em termos de lucro, o $\mathrm{B} \& \mathrm{~B}$ foi inferior à heurística em apenas $1,14 \%$, enquanto em termos de redução de TUPs, superou-a em 33 TUPs, que representa o $0,80 \%$ da quantidade total de TUPs. Uma explicação para esse fato é que havendo, na área de estudo, uma região de alta densidade de TUPs lucrativos, a forma de único passo de eliminação sequencial, que a heurística usa, impediu que a eliminação nessa área fosse mais radical do que o $\mathrm{B} \& \mathrm{~B}$. Na prática, porém, mesmo sem a restrição de número mínimo de TUPs, uma eliminação radical de TUPs não parece ser viável. Isto porque muitos dos TUPs ficariam com a capacidade totalmente utilizada, resultando em uma cobertura com pouca redundância para amenizar as consequências de panes e vandalismos, o que, certamente, causaria reclamações sobre a qualidade do serviço.

Os resultados acima indicam que qualquer dos dois métodos testados pode produzir, em tempo viável, resultados satisfatórios para estudos reais. Como o problema envolve muitas variáveis e restrições, é de se esperar um grande número de soluções ótimas e próximas de ótimas. Isso sugere que, em aplicações reais, o tempo computacional pode ser elevado, mas a qualidade das soluções, muito provavelmente, será satisfatória.

Tabela 1. Resultados dos testes na área total em estudo.

\begin{tabular}{|c|c|c|}
\hline Área total & Sem restrição de mínimo de TUPs & Com restrição de mínimo de TUPs \\
\hline Limitante de PL & $\mathrm{R} \$ 417.602,41$ & $\mathrm{R} \$ 378.642,49$ \\
\hline Número inicial de TUPs & 4.086 & 4.086 \\
\hline \multicolumn{3}{|l|}{ Solução por B\&B } \\
\hline Lucro máximo encontrado & $\mathrm{R} \$ 392.377,17$ & $\mathrm{R} \$ 377.783,26$ \\
\hline$\%$ Gap & $6,43 \%$ & $0,23 \%$ \\
\hline Tempo computacional & 86.504 segundos $(\approx 24$ horas $)$ & 86.438 segundos $(\approx 24$ horas $)$ \\
\hline TUPs mantidos & 849 & 1.517 \\
\hline$\%$ TUPs mantidos & $20,28 \%$ & $37,13 \%$ \\
\hline \multicolumn{3}{|c|}{ Solução factível do B\&B em tempo similar ao da heurística } \\
\hline Lucro máximo encontrado & $\mathrm{R} \$ 392.377,17$ & $\mathrm{R} \$ 373.275,24$ \\
\hline$\%$ Gap & $6,43 \%$ & $1,44 \%$ \\
\hline Tempo computacional & 22.595 segundos $(\approx 6$ horas 3 minutos $)$ & 13.056 segundos $(\approx 3$ horas 7 minutos $)$ \\
\hline \multicolumn{3}{|l|}{ Solução pela heurística } \\
\hline Lucro máximo encontrado & $\mathrm{R} \$ 396.903,83$ & $\mathrm{R} \$ 378.642,49$ \\
\hline$\%$ Gap & $5,22 \%$ & $0 \%$ \\
\hline Tempo computacional & 22.480 segundos $(\approx 6$ horas 3 minutos $)$ & 12.940 segundos $(\approx 3$ horas 6 minutos $)$ \\
\hline TUPs mantidos & 882 & 1.517 \\
\hline$\%$ TUPs mantidos & $21,59 \%$ & $37,13 \%$ \\
\hline
\end{tabular}




\subsection{Resultados com particionamento}

A Tabela 2 resume os resultados obtidos para a solução do problema com particionamento geográfico. $\mathrm{O}$ cuidado em fazer a partição segundo uma linha norte-sul que produzisse bordas com poucos TUPs fez com que o número inicial de TUPs na parte oeste (que possui maior densidade) ficasse bem maior do que na leste.

Como se pode ver da Tabela 2, a soma dos limitantes de programação linear (obtido pela relaxação da integralidade das variáveis $Y$ ) dá um valor de $\mathrm{R} \$ 417.327,04$, apenas $0,07 \%$ menor do que o obtido sem particionamento. Portanto, o particionamento feito sem que as subáreas fossem naturalmente disjuntas teve apenas um pequeno efeito no limitante para o ótimo.

$\mathrm{O}$ efeito do particionamento sobre o lucro obtido pela heurística foi insignificante ( $\mathrm{R} \$ 396.903,83$ e $\mathrm{R} \$ 396.900,41$ respectivamente, sem e com particionamento), enquanto o tempo para obtenção da solução com particionamento foi cerca de $56 \%$ menor do que o sem particionamento. Note-se, ainda, que o número de TUPs mantidos na solução por particionamento (883 TUPs) é, também, praticamente o mesmo da solução sem particionamento (882 TUPs). Observe-se que, mesmo em 24 horas de execução até a interrupção prematura, o B\&B não gerou soluções ótimas para os problemas das partições e que as soluções por ele produzidas são sensivelmente inferiores às da heurística.

O problema de "costura das bordas", isto é, de compatibilizar a cobertura e as alocações, ao menos nos testes feitos neste estudo, não parece ser empecilho se a linha de particionamento for feita em região de baixa densidade. Na solução obtida pela heurística, os TUPs mantidos e excluídos na solução com o particionamento foram, com duas exceções, os mesmos obtidos sem o particionamento.

Portanto, há uma indicação clara de que a técnica de particionamento pode ter um efeito substancial na redução do tempo computacional sem perda significativa no valor ótimo. Isso confirma a observação de que, em problemas de localização de grande porte, o particionamento geográfico é, com frequência, uma técnica eficaz para tornar o problema computacionalmente tratável.

\section{Discussão e conclusões}

Neste artigo, analisou-se o problema de redimensionamento de uma rede de telefones públicos, sua formulação e recursos simples para sua solução em escala real. Essa formulação foi traduzida num modelo de localização-atribuição com cobertura mínima e estrutura de programação linear inteira. Na prática, esse tipo de problema ocorre com certa frequência quando empresas fundem, ou passam a operar em conjunto, suas redes de postos de serviços (p.ex. caixas eletrônicos de bancos e postos de distribuição de gás doméstico).

O problema reúne alguns aspectos raros na literatura. Em primeiro lugar, ele trata da redução de instalações de

Tabela 2. Resultados para a área particionada em oeste e leste (sem restrição de mínimo de TUPs).

\begin{tabular}{|c|c|c|c|}
\hline Particionamento & Subárea oeste & Subárea leste & Área total \\
\hline Limitante de PL & $\mathrm{R} \$ 328.466,58$ & $\mathrm{R} \$ 88.860,46$ & $\mathrm{R} \$ 417.327,04$ \\
\hline Número inicial de TUPs & 2.616 & 1.470 & 4.086 \\
\hline \multicolumn{4}{|l|}{ Solução por B\&B } \\
\hline Lucro máximo encontrado & $\mathrm{R} \$ 313.524,09$ & $\mathrm{R} \$ 75.491,30$ & $\mathrm{R} \$ 389.015,39$ \\
\hline$\%$ Gap & $4,77 \%$ & $17,71 \%$ & $7,28 \%$ \\
\hline Tempo computacional & $\begin{array}{l}86.520 \text { segundos } \\
\quad(\approx 24 \text { horas })\end{array}$ & $\begin{array}{l}86.468 \text { segundos } \\
\quad(\approx 24 \text { horas })\end{array}$ & $\begin{aligned} & 172.988 \text { segundos } \\
(\approx & 48 \text { horas } 03 \text { minutos })\end{aligned}$ \\
\hline TUPs mantidos & 497 & 333 & 830 \\
\hline$\%$ TUPs mantidos & $19,00 \%$ & $22,65 \%$ & $20,31 \%$ \\
\hline \multicolumn{4}{|c|}{ Solução factível do B\&B em tempo similar ao da heurística } \\
\hline Lucro máximo encontrado & $\mathrm{R} \$ 313.524,09$ & $\mathrm{R} \$ 75.423,26$ & $\mathrm{R} \$ 388.947,35$ \\
\hline$\%$ Gap & $4,77 \%$ & $17,82 \%$ & $7,30 \%$ \\
\hline Tempo computacional & $\begin{aligned} & 9.579 \text { segundos } \\
(\approx 2 \text { horas } & 40 \text { minutos })\end{aligned}$ & $\begin{array}{c}681 \text { segundos } \\
(\approx 0 \text { horas } 11 \text { minutos })\end{array}$ & $\begin{aligned} & 10.260 \text { segundos } \\
(\approx & 2 \text { horas } 51 \text { minutos })\end{aligned}$ \\
\hline \multicolumn{4}{|l|}{ Solução pela heurística } \\
\hline Lucro máximo encontrado & $\mathrm{R} \$ 317.659,11$ & $\mathrm{R} \$ 79.241,30$ & $\mathrm{R} \$ 396.900,41$ \\
\hline$\%$ Gap & $3,40 \%$ & $12,14 \%$ & $5,15 \%$ \\
\hline Tempo computacional & $\begin{array}{c}9.233 \text { segundos } \\
(\approx 2 \text { horas } 33 \text { minutos })\end{array}$ & $\begin{array}{c}680 \text { segundos } \\
(\approx 0 \text { horas } 11 \text { minutos })\end{array}$ & $\begin{array}{c}9.913 \text { segundos } \\
(\approx 2 \text { horas } 45 \text { minutos })\end{array}$ \\
\hline TUPs mantidos & 523 & 360 & 883 \\
\hline$\%$ TUPs mantidos & $19,99 \%$ & $24,49 \%$ & $21,61 \%$ \\
\hline
\end{tabular}


serviço já existentes permitindo uma aplicação imediata, pois dados de demanda (ainda que aproximados) estão facilmente disponíveis. Além disso, trata-se de um problema de maximização de lucro e não de cobertura mínima ou de atendimento máximo a uma população.

Como frequentemente ocorre em problemas de localização, o porte que o problema assume em situações reais - e não sua singela formulação - constitui o maior impedimento para aplicações interessantes. Tendo como objetivo viabilizar essas aplicações, buscou-se uma forma de solução simples e de fácil implementação. Testes computacionais, com dados obtidos de uma situação real, foram feitos para avaliar a viabilidade de se utilizar um algoritmo de ramificação e limitação (B\&B) com interrupção prematura e, também, uma heurística de fácil implementação. Os resultados dos testes não são definitivos, pois a eficiência dos métodos é muito dependente dos dados, mas dão indicações claras da viabilidade de resolução de problemas em escala real por ambas as vias.

A decomposição do problema, através do particionamento geográfico, foi aqui explorada como uma forma de reduzir o esforço computacional e, nos testes feitos usando a heurística, mostrou-se promissor ao gerar resultados praticamente tão bons quanto os obtidos sem o particionamento e em tempo $56 \%$ menor. Tal particionamento é dificultado pela exigência de um total mínimo de terminais por habitante da área urbana, que estabelece uma ligação entre os subproblemas resultantes do particionamento da área. Entretanto, se o número de subáreas não for grande, não deve haver problema em se distribuir discricionariamente o total mínimo exigido de TUPs entre as subáreas. De fato, na prática, frequentemente existem razões de diversas naturezas para a empresa querer determinar a densidade de TUPs em cada subárea.

Alguns aspectos do problema foram apenas citados no presente estudo. Desses, um dos mais importantes foi a questão de confiabilidade do serviço e, por consequência, a necessidade de redundância na cobertura a ela associada. Telefones e outros equipamentos públicos são muito expostos a agressões de diversas naturezas, em particular, ao vandalismo. O reparo de telefones públicos é uma grande fonte de despesa e a degradação do serviço é sempre motivo de reclamações dos usuários. $\mathrm{O}$ modelo aqui desenvolvido é guiado apenas pela lucratividade e, consequentemente, tende a gerar soluções pouco recomendáveis em termos de redundância. Existem na literatura diversas sugestões (HOGAN; REVELLE, 1986; FIGUEIREDO et al., 2003) para tratar a questão de cobertura com redundância no contexto de localização de ambulâncias e outros serviços que exigem alta confiabilidade.

A incorporação, no modelo, do aspecto de confiabilidade do serviço ainda não foi explorada. Mas, algumas das idéias sugeridas na literatura poderiam ser tentadas no caso aqui tratado. Formas simples para tratar esse problema são discutidas a seguir:

a) alterar os dados, nas regiões onde se deseja maior confiabilidade, reduzindo-se a capacidade de atendimento dos TUPs ou a distância máxima (padrão) de cobertura. Essa alternativa, simples de implementar, talvez tenha o inconveniente de exagerar a redundância em algumas regiões mais densas e reduzi-la muito nas de baixa densidade. Além disso, não é fácil determinar que alterações devem ser feitas nos dados para se obter o efeito desejado; e

b) acrescentar restrições impondo que, na região onde se deseja redundância, cada TUP tenha em sua área de cobertura no mínimo tantos TUPs quanto desejado. Isto é, acrescentar ao modelo (1)-(6) restrições do tipo $\sum_{j \in A_{i}} Y(j) \geq \operatorname{Nred}(i)$ para todo TUP $i$ na região em que se deseje redundância, onde Nred(i) é o número mínimo de TUPs que se deseja na área de cobertura do TUP $i$. Como essas restrições poderiam tornar o problema infactível, ou reduzir muito a lucratividade, ao invés delas, poderiam ser acrescentadas as restrições $\sum_{j \in A_{i}} Y(j) \geq \operatorname{Nred}(i) Z(i)$ para cada TUP $i$ na região em que se deseje redundância, onde $\mathrm{Z}(\mathrm{i}) \in\{0,1\}$ é uma variável indicadora de redundância. Além disso, à função objetiva seria somado o termo ValRed(i) Z(i), em que ValRed(i) é o valor dado à redundância. É claro que tais alterações no modelo devem aumentar muito o tempo de resolução, que já é crítico, podendo inviabilizar o uso do modelo em problemas reais.

O tempo para obtenção de uma boa solução poderia ser mais explorado. Apesar de o tempo computacional ser viável, o desenvolvimento de novas heurísticas e, em particular, a tentativa de aplicação de meta-heurísticas se apresenta como possibilidade para prosseguimento da pesquisa na redução do tempo de resolução do modelo. Outro aspecto inexplorado nesta pesquisa foi a determinação da função de admitância, ou seja, a perda de receita que o fechamento de um TUP ocasiona. Para seu estudo, uma idéia seria usar dados históricos, i.e., examinar o que realmente ocorreu quando TUPs foram removidos; outra seria realizar entrevistas com usuários para quantificar suas percepções.

Finalmente, cabe ressaltar que, em muitas aplicações industriais, a programação matemática pode ser um importante auxílio à tomada de decisões, mas isso não significa que ela, por si só, seja capaz de apresentar uma solução a ser implementada. No presente caso, há muitas nuanças da realidade que não podem, ou não devem - por questão de dificuldade de implementação, de obtenção de dados ou pelo esforço computacional- ser incorporadas ao modelo. Em muitos desses aspectos, o melhor que se pode fazer 
para auxiliar os decisores é levar-lhes informação útil e na forma desejada, permitindo, assim, que usem seu julgamento e apliquem o conhecimento tácito adquirido com a experiência. Para este e qualquer problema envolvendo distribuição espacial, um grande auxílio nesse sentido é dado pelos sistemas de informação geográfica (GIS) como mostra Schaefer (2006). O modelo proposto neste artigo, principalmente quando combinado com o GIS, é, certamente, um auxílio importante para: (a) dar uma idéia clara do ganho potencial com a remoção de TUPs; (b) gerar uma proposta inicial de quais TUPs, dentre os milhares existentes, deverão ser removidos; e (c) estudar o efeito de remover determinados TUPs ou de acrescentar TUPs em determinados locais.

\title{
Resizing urban public telephone networks
}

\begin{abstract}
This paper discusses the problem of downsizing urban public telephone networks to improve the profitability of this business under the regulation imposed by the Agência Nacional de Telecomunicações (Brazilian National Telecommunication Agency). This study presents a pragmatic approach emphasizing the production of a good solution for companies. Peculiarities of the problem lead to the formulation of a location-allocation model with minimal coverage constraints which, in actual scale, is too large for exact solution by means of conventional mixed integer programming methods. This leads to examining the feasibility of obtaining a good solution avoiding the use of complex methods of difficult implementation. Possible ways of reducing and partitioning the problem are discussed as well as their solution by means of premature interruption of a branch and bound algorithm and also by means of a simple and easy to implement heuristics. Results of a computational test are presented and indicate the feasibility of industrial applications of the model.
\end{abstract}

Keywords: Public telephones. Integer linear programming. Location-allocation. Coverage constraints. Heuristic.

\section{Referências Bibliográficas}

BISSCHOP, J.; ROELOFS, M. AIMMS - The User's Guide. Haarlem: Paragon Decision Technology BV. 1999. 232 p.

BRANDEAU, M. L.; CHIU, S. S. An overview of representative problems in location research. Management Science, v. 35, n. 6, p. 645-674, jun 1989.

BRASIL. Decreto n ${ }^{\circ} 2.592$, de 15 de maio de 1998. Aprova o Plano Geral de Metas para a Universalização do Serviço Telefônico Fixo Comutado Prestado no Regime Público. Disponível em: <http://www.planalto.gov.br/ccivil_03/decreto/D2534.htm>. Acesso em: 3 abr 2006.

DREZNER, T.; DREZNER, Z. Replacing continuous demand with discrete demand in a competitive location model. Naval Research Logistics, v. 44, n. 1, p. 81-95, 1997.

DREZNER, Z.; WESOLOWSKY, G. O.; DREZNER, T. On the logit approach to competitive facility location. Journal of Regional Science, v. 38, n.2, p. 313-327, 1998.

FIGUEIREDO, A. P. S.; LORENA, L. A. N.; DE CARVALHO, S. V. Modelos de localização de ambulâncias. III WORCAP, 2003, São José dos Campos. São José dos Campos, SP: INPE, 2003. 6 p.

GALVÃO, R. D.; CHIYOSHI, F. Y.; MORABITO, R. Towards unified formulations and extensions of two classical probabilistic location models. Computers \& Operations Research, v.32, n. 1, p. 15-33, 2005.

HALE, T. S.; MOBERG, C. R. Location science research: A review. Annals of Operations Research, v.123, n. 1-4, p. 21-35, out 2003.

HOGAN, K.; REVELLE, C. S. Concepts and applications of backup coverage. Management Science, v.32, n. 11, p. 1434-1444, 1986.

MARIANOV, V.; SERRA, D. Probabilistic, maximal covering location-allocation models for congested systems. Journal of Regional Science, v. 38, n. 3, p 401-424, ago 1998.

PERALES, R. C. Consumer choice in competitive location models. Barcelona, 2002. 159 p. Tese de Doutorado - Department d'Economia i Empresa, Universitat Pompeu Fabra.

SCHAEFER, D. M. Melhorando a rentabilidade de uma rede de telefonia pública usando sistema de informação geográfica. Rio de Janeiro, 2006. 56 p. Dissertação de mestrado - Departamento de Engenharia Industrial, Pontifícia Universidade Católica do Rio de Janeiro.

WORKSHOP DE PARCERIA UNICAMP \& TELEFÔNICA, FASE II, 2004, Campinas. Caderno de resumos... Campinas: UNICAMP, julho de 2004. 32 p. 


\section{Sobre os autores}

\section{Leonardo Junqueira Lustosa}

Departamento de Engenharia Industrial, Pontifícia Universidade Católica do Rio de Janeiro - PUC-Rio,

Rio de Janeiro, RJ, Brasil,

e-mail: lj!@ ind.puc-rio.br

\section{Cristina de las Nieves Araneda-Fuentes}

Departamento de Ingeniería, Universidad Arturo Prat - UNAP,

Iquique, Chile,

e-mail: caraneda@daad-alumni.de

\section{Daniel de Mello Schaefer}

Departamento de Engenharia Industrial, Pontifícia Universidade Católica do Rio de Janeiro - PUC-Rio, Rio de Janeiro, RJ, Brasil,

e-mail: daniel.schaefer@telemar.com.br

Agradecimentos. Os autores agradecem à empresa que forneceu os dados, à Paragon Decision Technology pela licença acadêmica do AIMMS e apoio técnico. Agradecem, também, aos professores Eugênio K. Epprecht, Madiagne Diallo e a dois revisores anônimos pelas críticas e sugestões que muito melhoraram o artigo. Reconhecem, ainda, o apoio financeiro dado pela CAPES através da bolsa de doutorado concedida à autora.

Recebido 1/11/2006

Aceito 11/2/2009 